\title{
Dynamic models of the sun from the convection zone to the chromosphere
}

\author{
Sven Wedemeyer-Böhm ${ }^{1}$ \\ ${ }^{1}$ Kiepenheuer-Institut für Sonnenphysik, Schöneckstr. 6, 79104 Freiburg, Germany \\ email: wedemeyer@kis.uni-freiburg.de
}

\begin{abstract}
The chromosphere in internetwork regions of the quiet Sun was regarded as a static and homogeneous layer for a long time. Thanks to advances in observations and numerical modelling, the wave nature of these atmospheric regions received increasing attention during the last decade. Recent three-dimensional radiation magnetohydrodynamic simulations with CO5BOLD feature the chromosphere of internetwork regions as a dynamic and intermittent phenomenon. It is a direct product of interacting waves that form a mesh-like pattern of hot shock fronts and cool post-shock regions. The waves are excited self-consistently at the top of the convection zone. In the middle chromosphere above an average height of $1000 \mathrm{~km}$, plasma beta gets larger than one and magnetic fields become more important. The model chromosphere exhibits a magnetic field that is much more homogeneous than in the layers below and evolves much faster. That includes fast propagating (MHD) waves. Further improvements of the simulations like time-dependent hydrogen ionisation are currently in progress. This class of models is capable of explaining apparently contradicting diagnostics such as carbon monoxide and UV emission at the same time.
\end{abstract}

Keywords. Convection, hydrodynamics, MHD, radiative transfer, shock waves, Sun: chromosphere, granulation, photosphere, radio radiation, UV radiation.

\section{Introduction}

The atmosphere of the Sun is a very dynamic and inhomogeneous layer that exhibits phenomena on a large range of spatial and temporal scales. A self-consistent and comprehensive numerical model that can match all accessible chromospheric diagnostics would be of great value. The complexity of the object, however, so far limited all efforts to more or less crude approximations or at least to addressing certain aspects only. Modelling of these layers is numerically very demanding since a realistic description requires the inclusion of many physical ingredients. A three-dimensional and time-dependent modelling is mandatory (cf. Carlsson \& Stein 1995; Skartlien et al. 2000; Stein \& Nordlund 1989, 2006; and many more). In order to make progress, the radiation magnetohydrodynamics code CO $^{5}$ BOLD (Freytag et al. 2002) has been upgraded recently. It now offers a large range of possible applications, including the time-dependent treatment of chemical reaction networks, hydrogen ionisation, and magnetic fields.

This paper addresses the potential of the currently possible radiation magnetohydrodynamics simulations and the synthesis of intensity maps for interpreting observations and even defining constraints for future observation campaigns for internetwork regions of the Sun. To demonstrate this, a new three-dimensional model is used as input for the calculation of synthetic intensity images. 


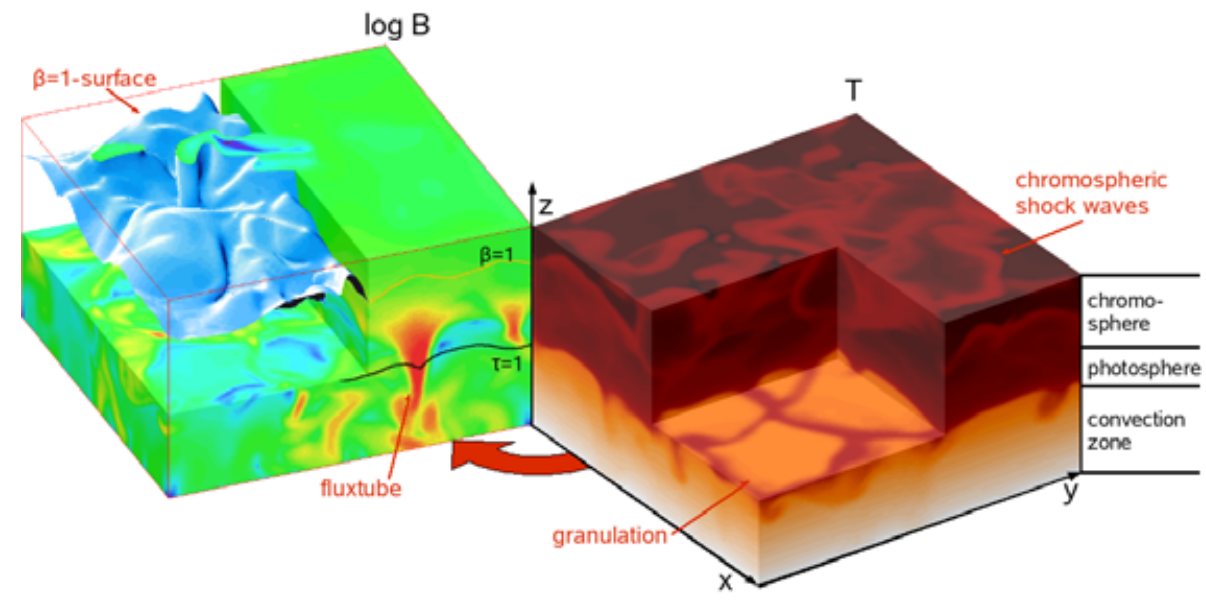

Figure 1. Three-dimensional magnetohydrodynamics model: Gas temperature $T$ (right cube)

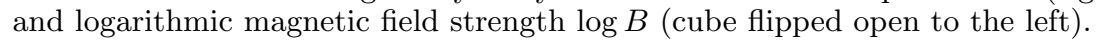

\section{Time-dependent radiation (magneto-)hydrodynamics}

The numerical simulations presented here were all carried out with an upgraded version of CO5 BOLD (Freytag et al. 2002, Wedemeyer et al. 2004, Schaffenberger et al. 2005), a radiation magnetohydrodynamics code for simulating the convective layers and atmospheres of stars. Additional features are the time-dependent treatments of dust formation (Höfner et al. 2004), chemical reaction networks (Wedemeyer-Böhm et al. 2005, 2006), and also non-equilibrium hydrogen ionisation (Leenaarts \& Wedemeyer-Böhm 2006a, 2006b, hereafter L06). Radiative cooling due to spectral lines of carbon monoxide can now be taken into account (Wedemeyer-Böhm \& Steffen submitted, hereafter W06). Operator splitting allows to treat hydrodynamics, tensor viscosity, chemistry, non-equilibrium hydrogen ionisation, and radiative transfer in subsequent steps of this order. The overall computational time step is typically around $0.1 \mathrm{~s}$ to $0.2 \mathrm{~s}$ for non-magnetic simulations but up to a factor ten or more smaller for the MHD case, depending on magnetic field strength. The lateral boundary conditions are periodic whereas the lower boundary is "open", i.e. material can flow in and out of the computational box. The upper boundary condition is usually of 'transmitting' type so that shock waves can leave the computational domain without being reflected.

\section{The numerical model}

The new 3D model presented here (see Fig. 1) is similar to the model by Wedemeyer et al. (2004, hereafter W04) and Schaffenberger et al. (2005, hereafter S05) but was calculated with non-grey radiative transfer (multi-group with ATLAS6 opacities, Kurucz 1970) and time-dependent CO chemistry with the same chemical reaction network as used by Wedemeyer-Böhm et al. (2005). With a horizontal extent of $4800 \mathrm{~km} \times 4800 \mathrm{~km}$ (corresponding to $\approx 6^{\prime \prime} .6 \times 6^{\prime \prime} .6$ in ground-based observations) it is slightly smaller than the model by W04. It reaches from $z=-1400 \mathrm{~km}$ in the convection zone to the middle chromosphere at a height of $z \sim 1700 \mathrm{~km}$. The origin of the geometric height scale $(z=0 \mathrm{~km})$ is adjusted to the average Rosseland optical depth unity. The photosphere is here referred to as the layer between $0 \mathrm{~km}$ and $500 \mathrm{~km}$ in model coordinates, and the chromosphere as the layer above. The spatial resolution is $\Delta x=\Delta y=40 \mathrm{~km}$ in horizontal directions, whereas the grid cells are non-equidistantly spaced in vertical direction 
( $\Delta z=46 \mathrm{~km}$ at the bottom, decreasing with height to $12 \mathrm{~km}$ for $z \geqslant-270 \mathrm{~km}$ ). The treatment of radiative transfer is switched to grey for the chromosphere. The initial field has a strength of $B_{0}=10 \mathrm{G}$ only and is vertical and unipolar.

\section{Structure and dynamics of the model atmosphere}

The new model is very similar to recent other models of the $\mathrm{CO}^{5} \mathrm{BOLD}$ group concerning atmospheric structure and dynamics of solar internetwork regions (see W04, S05, L06 etc.). Altogether the simulations yield a new picture of the solar atmosphere in internetwork regions as highly structured, intermittent, and dynamic phenomenon. A key role play the ubiquitous propagating and interacting shock waves and adiabatically expanding post-shock regions that produce an intermittent mix of co-existing hot and cool regions in the chromosphere. But also the photosphere below exhibits structure on very small scales. The reversed granulation pattern in the middle photosphere provides cool regions where the largest absolute amount of carbon monoxide molecules is located. The panels $\mathrm{a}$ and $\mathrm{b}$ of Fig. 2 show gas temperature and $\mathrm{CO}$ number density in the middle photosphere at $z=210 \mathrm{~km}$, respectively. Gas temperature and CO number density are closely anti-correlated (-0.86), owing to the strong temperature sensitivity of carbon monoxide. The cool areas in the middle photosphere allow for formation of more CO compared to the hotter "filamentary" regions by typically 1-2 orders of magnitude. But CO is also very abundant in the (low) chromosphere above and binds a large fraction of all available carbon atoms there. An exception are shocks where CO is significantly depleted by dissociation. The distribution of $\mathrm{CO}$ with a peak in absolute number density on average at $z=150 \mathrm{~km}$ is in line with the findings by Uitenbroek (2000a,b) and Asensio Ramos et al. (2003). The shock waves also hamper the cooling action of infrared CO lines. Due to the continuous passage of these waves the atmosphere cannot relax to a cool state (W06). The average temperature is only reduced by $\sim 100 \mathrm{~K}$.

In sharp contrast to the thermal structure of the model chromosphere, the ionisation degree of hydrogen is rather homogeneous in the upper layers at values set by the passing hot wave fronts (L06). A similar picture is found for the magnetic field in the chromosphere of internetwork regions that is much more homogeneous and also more dynamic than in the layers below (S05). The highly dynamic middle chromosphere seems to be separated from the slower evolving lower layers by the surface of plasma $\beta=1$ (at an average height of $z=1000 \mathrm{~km}$ ). The photospheric field is highly structured with a kind of "small-scale canopy" above granule interiors as a result of flux expulsion by the hydrodynamic flow. Even a weak initial field of $10 \mathrm{G}$ is sufficient to build up $\mathrm{kG}$ flux tubes whereas the field strength above the granule interiors often drops to only $\sim 3 \mathrm{G}$. This finding is still compatible with observations by Trujillo Bueno et al. (2004) using the Hanle effect (see S05).

\section{Synthetic intensity maps}

A snapshot from the 3D simulation described above is used as input for radiative transfer calculations with Linfor3D (http://www.aip.de/ mst/linfor3D_main.html) for a small selection of spectral features: The line wing of $\mathrm{CaIIH}$ at 369.7,nm and the continuum at wavelengths of $160 \mathrm{~nm}, 500 \mathrm{~nm}$, and $1 \mathrm{~mm}$ (see Fig. 2). All intensity maps refer to disk-centre.

The continuum intensity at a wavelength of $500 \mathrm{~nm}$ (Fig. 2e) clearly exhibits the granulation pattern in an internetwork region (i.e. with weak magnetic field only). It has an 


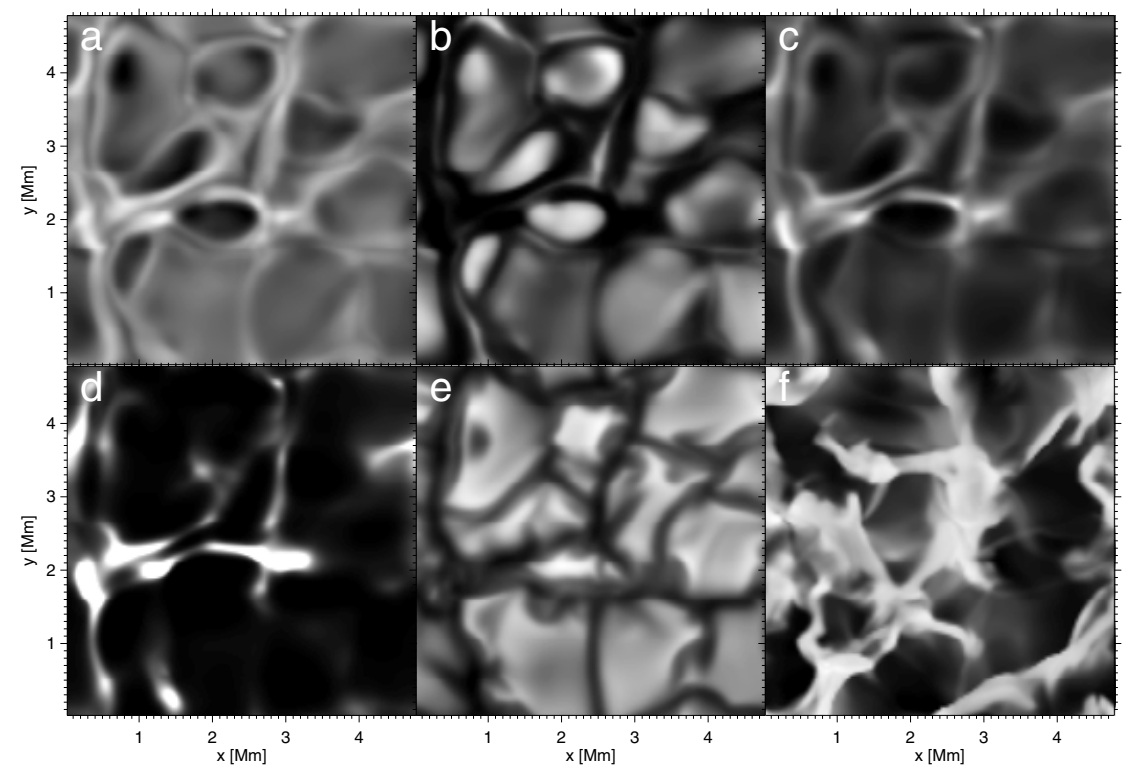

Figure 2. Non-grey three-dimensional model: (a) Gas temperature at a height of $z=220 \mathrm{~km}$, (b) $\mathrm{CO}$ number density at the same height, (c) emergent intensity at $396.7 \mathrm{~nm}$ in the line wing of $\mathrm{CaII} \mathrm{H}$, and in the continuum at $(d) 160 \mathrm{~nm},(e) 500 \mathrm{~nm}$, and $(f) 1 \mathrm{~mm}$ (grey RT). The data range of panel $\mathrm{d}$ is limited to $30 \%$ of its maximum value.

intensity contrast of $\delta I_{\mathrm{rms}} /\langle I\rangle=22 \%$, whereas the corresponding contrast of the emergent grey intensity is $17 \%$ and thus the same as for the 3D hydrodynamic model with grey radiative transfer by W04.

The Ca II H line wing intensity (Fig. 2c, see also Leenaarts \& Wedemeyer-Böhm 2005) originates from the middle photosphere and thus shows the same although reversed pattern as the $\mathrm{CO}$ distribution (panel b). The contrast is $41 \%$ and thus much higher than for the $500 \mathrm{~nm}$ continuum. The intensity at $160 \mathrm{~nm}$ is on average formed only a little bit higher in the high photosphere. In contrast to the Ca II H line wing map, the intensity image at $160 \mathrm{~nm}$ only exhibits the hottest regions of the reversed granulation pattern as the gas temperature translates highly non-linearly into intensity at UV wavelengths. Most of the pattern appears relatively dark and is thus hard to detect so that the hot regions resemble isolated grainy features. The contrast is quite extreme and reaches $190 \%$ for the displayed map. Already the resolution of $0.5^{\prime \prime}$ (or more) of the $160 \mathrm{~nm}$ channel of the Transition Region and Coronal Explorer (TRACE, Handy et al. 1999; Schneider et al. 2004) might be too low for detecting the faint background pattern. In combination with $\mathrm{CO}$ and $\mathrm{Ca}$ line wing diagnostics, the UV continuum is nevertheless a valuable atmospheric probe. The middle photosphere can also be observed in the cores of spectral lines as, e.g., Fe I at $709.04 \mathrm{~nm}$ (Janssen \& Cauzzi 2006).

The situation is more complicated for the solar chromosphere as (i) meaningful diagnostics are rare and/or hard to interpret and (ii) realistic multi-dimensional numerical modelling of this layer is difficult due to many reasons and hardly possible with nowadays computational resources. The central parts of the CaII resonance lines are commonly used as chromospheric diagnostic (see Wöger et al. 2006 for a recent example) but are difficult to interpret as the intensity has contributions from a large height range and non-local/non-equilibrium effects have to be taken into account. In contrast, the gas temperature almost linearly translates into continuum intensity in the (sub-)millimetre 
range (see Fig. 2f for a continuum intensity map at $1 \mathrm{~mm}$ ). Unfortunately the current instruments are not able to resolve the small spatial scales discussed here (see, e.g., White et al. 2006). The future Atacama Large Millimeter Array (ALMA, Beasley et al. 2006), a interferometer with a large number of antennae in the (sub-)millimetre range, will provide sufficiently high spatial resolution for a detailed comparison with numerical models. The receivers will cover a wavelength range from $0.3 \mathrm{~mm}$ to $9 \mathrm{~mm}$. The intensity at the different wavelengths originates from different layers from the high photosphere to the middle chromosphere, with the average formation height increasing with wavelength. Simultaneous observation at different wavelengths could thus serve as a tomography of the atmosphere, finally allowing for mapping the (three-dimensional) thermal structure of the solar chromosphere (Loukitcheva et al. 2006; Wedemeyer-Böhm et al. in prep.). The intensity contrast at $1 \mathrm{~mm}$ is $23 \%$ and thus very similar to the $500 \mathrm{~nm}$ continuum.

\section{Discussion and Conclusions}

Despite the steady increase in realism many details of the models still have to be taken with caution. Maybe the strongest limitation is the assumption of LTE (in particular for the radiative transfer) which was necessary to keep the problem computationally tractable so far. LTE is also assumed in the "post-simulation" radiative transfer calculations with Linfor3D. The extension to a more realistic non-LTE treatment in both the simulation and the intensity/spectrum synthesis will be an important point to work on in future. Consequently, the temperature amplitudes of the current model chromosphere might still be uncertain to some extent.

Nevertheless this class of models can give important hints for the interpretation of observations. Co-existing cool and hot regions in an inhomogeneous and dynamic atmosphere offer the ultimate solution for the diagnostic dilemma with apparently contradicting temperatures derived from $\mathrm{CO}$ on the one and UV-based diagnostics on the other hand. These different observational aspects can now be explained together with one single model, like the one presented here, whereas conventional (semi)-empirical 1D models (e.g., Vernazza et al. 1981) can at best account for selected aspects of the atmosphere. The full potential of detailed 3D models gets obvious when addressing the small-scale spatial pattern. For instance, the models imply that the spatial resolution of TRACE at $160 \mathrm{~nm}$ is not sufficient to resolve the small-scale pattern of the middle to upper photosphere. That has consequences for the conclusions derived for atmospheric dynamics and heating (Fossum \& Carlsson 2005).

A comprehensive numerical model is also helpful for defining constraints on diagnostics (in combination used as a kind of tomography) that are required for the desired empirical determination of the thermal structure of the atmosphere. For instance, the continua at (sub-)millimetre wavelengths as chromospheric diagnostics seem to be a promising alternative to the complicated inner regions of the Ca II resonance lines.

\section{Acknowledgements}

I would like to thank my colleagues B. Freytag, M. Steffen, H.-G. Ludwig, I. Kamp, J. Leenaarts, O. Steiner, J. Bruls, and R. Schlichenmaier for their contributions to various related projects and for many inspiring discussions. SW was supported by the Deutsche Forschungsemeinschaft (DFG), project Ste 615/5.

\section{References}

Asensio Ramos, A., Trujillo Bueno, J., Carlsson, M. \& Cernicharo, J. 2003, ApJL 588, L61 
Carlsson, M. \& Stein, R. F. 1995, ApJL 440, L29

Fossum, A. \& Carlsson, M. 2005, Nature 435, 919

Freytag, B., Steffen, M. \& Dorch, B. 2002, Astron. Nachr. 323, 213

Handy, B. N., Acton, L. W., Kankelborg, C. C., et al. 1999, Solar Phys. 187, 229

Höfner, S., Gautschy-Loidl, R., Aringer, B., et al. 2004, in: H.U. Käufl, R. Siebenmorgen, A. Moorwood (eds.), ESO Workshop "High Resolution Infrared Spectroscopy in Astronomy", Springer, $11 \mathrm{P}$

Janssen, K. \& Cauzzi, G. 2006, A\&A 450, 365

Kurucz, R. L. 1970, SAO Special Report 308

Leenaarts, J. \& Wedemeyer-Böhm, S. 2005, A\& $A$ 431, 687

Leenaarts, J. \& Wedemeyer-Böhm, S. 2006, in: J. Leibacher, H. Uitenbroek, and R. Stein (eds.), Proc. of NSO Workshop No. 23, in press

Leenaarts, J. \& Wedemeyer-Böhm, S., A\&AA, in press (L06)

Loukitcheva, M., Solanki, S. K. \& White, S. 2006, A\&\&A 456, 713

Schaffenberger, W., Wedemeyer-Böhm, S., Steiner, O. \& Freytag, B. 2005, in: D. E. Innes, A. Lagg \& S. A. Solanki (eds.), ESA SP-596: Chromospheric and Coronal Magnetic Fields, $65(\mathrm{~S} 05)$

Schneider, G., Pasachoff, J. M. \& Golub, L. 2004, Icarus 168, 249

Skartlien, R., Stein, R. F. \& Nordlund, Å. 2000, ApJ 541, 468

Stein, R. F. \& Nordlund, A. 1989, ApJL 342, L95

Stein, R. F. \& Nordlund, Å. 2006, ApJ 642, 1246

Trujillo Bueno, J., Shchukina, N. \& Asensio Ramos, A. 2004, Nature 430, 326

Uitenbroek, H. 2000a, ApJ 531, 571

Uitenbroek, H. 2000b, ApJ 536, 481

Vernazza, J. E., Avrett, E. H. \& Loeser, R. 1981, ApJS 45, 635

Wedemeyer, S., Freytag, B., Steffen, M., Ludwig, H.-G. \& Holweger, H. 2004, A\&A 414, 1121 (W04)

Wedemeyer-Böhm, S., Kamp, I., Bruls, J. \& Freytag, B. 2005, A\&A 438, 1043

Wedemeyer-Böhm, S. \& Steffen, M., submitted to $A \mathscr{E} A$

Wedemeyer-Böhm, S., Ludwig, H.-G., Steffen, M., Freytag, B. \& Leenaarts, J., in prep

White, S. M., Loukitcheva, M. \& Solanki, S. K. 2006, A\&A 456, 697

Wöger, F. Wedemeyer-Böhm, Schmidt, W. \& von der Lühe, O., $A \mathscr{G} A$, in press

\section{Discussion}

KUPKA: Have you looked at the effects of different upper boundary conditions on your simulations?

WEDEMEYER-BÖHM: Yes. We use a transmitting upper boundary and checked its influence for the hydrodynamics case and found no (artificial) reflection of waves (as it would be the case for a closed boundary). The further development of the boundary conditions for the MHD case is in progress but the current version already seems to work properly. There will be a new open lower and a transmitting upper MHD boundary condition available soon which will be checked thoroughly.

Martinez Pillet: What happens with the CO clouds, if you start with mean fields larger than $10 \mathrm{G}$ ?

WEDEMEYER-BÖHM: We have carried out 2D simulations with different field strengths which show a clear difference in the distribution of molecules (Wedemeyer-Böhm et al. 2005, ESA SP-596, 117). For a systematic study, however, we would like to compute a sequence of $3 \mathrm{D}$ models first. 